\title{
Paving the way: the road to be taken for proper NAFLD health care
}

\author{
A new Consensus Statement calls for national and global action bringing fatty liver disease to the \\ forefront of the public health-care system.
}

Robert Frost in his famous poem The Road Not Taken urges us to follow the less travelled path; this approach is generally great advice for researchers, but there are often moments in the history of science in which the road needs some pavement for more people to follow.

Nonalcoholic fatty liver disease (NAFLD) is a spectrum of chronic liver disease characterized by increased intrahepatic triglyceride accumulation, and it ranges from simple steatosis to steatosis in the presence of inflammation and of hepatocyte injury (nonalcoholic steatohepatitis (NASH)), and often in the presence of fibrosis that will eventually lead to cirrhosis ${ }^{1-3}$. Because of the complex immunological liver landscape combined with metabolic alterations and oxidative stress, treatment stratification is challenging, especially for patients with NAFLD-associated hepatocellular carcinoma (HCC).

NAFLD is a non-communicable disease (NCD) closely related to type 2 diabetes mellitus (T2DM) and obesity. According to the World Health Organization, NCDs are responsible for almost $70 \%$ of deaths globally. Despite NAFLD being a potentially serious chronic liver condition with NASH being a leading cause for HCC development ${ }^{1,2}$, it is not included in the current NCD care strategies.

A multidisciplinary group of 33 experts, and a wider consortium of $>200$ members, were gathered to address NAFLD burden, raise public awareness and highlight the necessity for proper disease management in a new Consensus Statement ${ }^{4}$. From December 2020 to March 2021, a group of clinicians, researchers, advocates, academics and civil society experts from 18 countries led a Delphi study to develop consensus statements and recommendations to improve NAFLD health-care strategies for the public ${ }^{4}$.

With obesity on the rise, the global prevalence of NAFLD among adults is estimated to be $23-25 \%$, whereas in children and adolescents the prevalence is estimated to be $7.6-34.2 \%$. Similar to adults, NAFLD prevalence is higher in children with obesity than in those without. The same scenario is observed in patients with T2DM: NAFLD prevalence is higher among adults and children with T2DM, and children with T2DM have a more aggressive NAFLD phenotype than adults.
Thus, more research is necessary to elucidate the pathophysiology of paediatric NAFLD ${ }^{4}$.

Unavoidably, the NAFLD burden has economic implications. Strong voices of the scientific community have already raised their concerns about the devastating financial impact the disease carries ${ }^{5,6}$. The authors of this Consensus Statement underline the necessity of gathering more data on the epidemiology and the financial strain of NAFLD, the lack of which constrains any efforts to improve the public health-care system. Moreover, a specific and defined model of care system, which to date has received very little attention, is vital for the management of the disease ${ }^{4}$.

And yet, despite the severe effects NAFLD has on health, quality of life and socioeconomic structures, the disease remains largely unknown to the non-specialist audience. To increase awareness, this new Consensus Statement calls for health-care professionals and policy-makers to communicate the risk factors and the potential consequences to the general public in simple, non-stigmatizing language. In addition, the liver health community ought to increase clarity and agree upon terminology. An area of current debate, for example, is the possible replacement of 'NAFLD' with 'metabolic dysfunction-associated fatty liver disease' (MAFLD) ${ }^{4}$.

This Consensus Statement - with endorsement from $>100$ global stakeholders including EASL, APASL, AASLD and EASO - scaffolds the frame under which global agreement on NAFLD management can be reached $^{4}$. We move forward carrying a common vision for the enhancement of NAFLD health care towards treatment improvement and prevention.

1. Anstee, Q. M. et al. From NASH to HCC: current concepts and future challenges. Nat. Rev. Gastroenterol. Hepatol. 16, 411-428 (2019).

2. Ringelhan, M. et al. The immunology of hepatocellular carcinoma. Nat. Immunol. 19, 222-232 (2018).

3. Younossi, Z. M. Non-alcoholic fatty liver disease - a global public health perspective. J. Hepatol. 70, 531-544 (2019).

4. Lazarus, J. V. et al. Advancing the global public health agenda for NAFLD: a consensus statement. Nat. Rev. Gastroenterol. Hepatol. https://doi.org/10.1038/s41575-021-00523-4 (2021).

5. Abdelmalek, M. F. The clinical and economic burden of NAFLD: time to turn the tide. Nat. Rev. Gastroenterol. Hepatol. 13, 685-686 (2016).

6. Hagström, H. et al. Health care costs of patients with biopsy-confirmed nonalcoholic fatty liver disease are nearly twice those of matched controls. Clin. Gastroenterol. Hepatol. 18, 1592-1599 (2020). 